\title{
ON THE RELIABILITY OF A RENEWABLE MULTIPLE COLD STANDBY SYSTEM
}

\author{
E. J. VANDERPERRE
}

Received 14 July 2004 and in revised form 10 December 2004

We present a general reliability analysis of a renewable multiple cold standby system attended by a single repairman. Our analysis is based on a refined methodology of queuing theory. The particular case of deterministic failures provides an explicit exact result for the survival function of the duplex system.

\section{Introduction}

Apart from important but particular cases, such as state-dependent exponential distributions, for example, [1,4] or an arbitrary repair time distribution [12], the reliability analysis of multiple cold standby systems is far from complete.

We present a general reliability analysis of a basic multiple cold standby system attended by a single repairman, henceforth called a $K$-system. The $K$-system consists of a single operative unit (the on-line unit) endowed with $K$ units in cold standby. The system acts as a closed queueing system evolving in time, that is, any failed unit is immediately replaced by a standby spare (if available upon failure) and taken into repair provided that the repairman is idle. Otherwise, the failed unit has to queue for repair. On the other hand, a repaired unit lines up in standby or becomes instantaneously operative if the $K$ remaining units are down. Our $K$-system is the statistical dual of the $K$-system described in [12]. An example of the so-called duplex system $(K=1)$ is the (internal) cooling device of the International Space Station (ISS) sustained by an (external) cooling device in cold standby. Note that astronauts can provide local repair.

Our $K$-system satisfies the usual conditions, that is, independent identically distributed random variables, perfect repair and queuing. Each operative unit has an arbitrary lifetime (failure-free time) distribution, a zero failure rate in standby (the so-called cold standby [2]), and a constant repair rate [8]. Note that our particular assumptions are less general with regard to the previous assumptions proposed in $[1,4]$.

But, on the other hand, our present assumptions are less restrictive since we are dealing with an arbitrary lifetime distribution.

In order to derive the survival function of the $K$-system, we first introduce a stochastic process defined on some filtered probability space $\{\Omega, B, \mathbf{P}, \mathfrak{F}\}$. Moreover, we define 
a suitable stopping time $\tau_{K}$, called the first system-down time. Then, we derive a straightforward relationship between the Laplace-Stieltjes-transforms $\mathbf{E} e^{-s \tau_{K}}$ and $\mathbf{E} e^{-s \beta_{K}}$, where $\beta_{K}$ denotes the busy period of the queuing system $M|G| 1 \mid K$ (see Cohen [6] for a precise description).

Finally, as an example, we derive the exact explicit survival function of the duplex system in the particular case of deterministic failures.

\section{Formulation}

Consider the basic $K+1$-unit cold standby system attended by a single repairman, henceforth called the $K$-system. Our $K$-system is the statistical dual of the $K$-system described in [12]. The $K$-system satisfies the usual conditions (i.i.d. random variables, perfect repair, queuing). The set of $K$ standby units is usually called a socket. Each operative unit has an arbitrary lifetime distribution $F(\cdot), F(0)=0$, a zero failure rate in standby (cold standby), and a constant repair rate $\lambda>0$. The Laplace-Stieltjes-transform of $F(\cdot)$ is denoted by $f(s)$, Res $\geq 0$. Let $X_{t}$ be the number of failed units in the repair shop at time $t$, so that $X_{t} \in\{0, \ldots, K+1\}$. The non-Markovian stochastic process $\left\{X_{t}, t \geq 0\right\}$ is defined on a filtered probability space $\{\Omega, B, \mathbf{P}, \mathfrak{F}\}$, where the history $\mathfrak{F}:=\left\{\mathfrak{F}_{t}, t \geq 0\right\}$ satisfies the Dellacherie conditions

(i) $\mathfrak{F}_{0}$ contains the $\mathbf{P}$-null sets of $B$,

(ii)

$$
\forall t \geq 0, \quad \mathfrak{F}_{t}=\bigcap_{u>t} \mathfrak{F}_{u},
$$

that is, the family $\mathfrak{F}$ is right continuous.

Consider the $\mathfrak{F}$-stopping time

$$
\tau_{K}:=\inf \left\{t>0: X_{t}=K+1 \mid X_{0}=1\right\}
$$

In reliability engineering, $\tau_{K}$ is usually called the first system-down time. The origin of time is fixed at the instant of the first failure, so that the busy period of the repairman starts at $t=0$, that is, $X_{0}=1, \mathbf{P}$-a.s. Finally, let

$$
\mathbb{R}^{(K)}(t):=\mathbf{P}\left\{\tau_{K}>t\right\}, \quad t \geq 0
$$

The tail $\mathbb{R}^{(K)}(\cdot)$ is called the survival function of the $K$-system.

\section{A preliminary result}

We are going to determine $\mathbf{E} e^{-s \tau_{K}}$ by means of a theorem due to Cohen [5] and Vanderperre [10]. Let $D_{\omega}$ be a circle at the origin of the complex $\omega$-plane, with counterclockwise orientation and with radius $|\omega|<|\rho(s)|$, where $\rho(s)$ is the smallest root of the functional equation $\omega=f(s+\lambda-\lambda \omega)$. Note that $\rho(s)=\mathbf{E} e^{-s \beta}$, where $\beta$ denotes the busy period of the queuing system $M|G| 1 \mid \infty$. See Lemma of Takács, for example, [6, Appendix]. 
Theorem $3.1[5,10]$. For $k \geq 1$ and $\operatorname{Re} s \geq 0$,

$$
\mathbf{E} e^{-s \beta_{k}}=\frac{1-(1-f(s))(1 / 2 \pi i) \int_{D_{\omega}}(1-\omega)^{-1}(\omega-f(s+\lambda-\lambda \omega))^{-1}\left(d \omega / \omega^{k-1}\right)}{1-(1-f(s))(1 / 2 \pi i) \int_{D_{\omega}}(1-\omega)^{-1}(\omega-f(s+\lambda-\lambda \omega))^{-1}\left(d \omega / \omega^{k}\right)}
$$

\section{The survival function}

It is plain that $\tau_{K}$ does not depend on the queuing order in the socket! Therefore, without loss of generality, we may assume (nonbreak-in) overall priority in operation for any repaired unit (if any). Consequently, taking the Markov property of the exponential distribution into account,

$$
\tau_{K} \stackrel{D}{=} \sum_{k=1}^{K} \beta_{k}, \quad K \geq 1
$$

It should be noted that the random variables $\beta_{k}, k=1, \ldots, K$, are statistically independent. Hence,

$$
\mathbf{E} e^{-s \tau_{K}}=\prod_{k=1}^{K} \mathbf{E} e^{-s \beta_{k}}
$$

By Theorem 3.1 and the identity

$$
\frac{1}{2 \pi i} \int_{D_{\omega}}(1-\omega)^{-1}(\omega-f(s+\lambda-\lambda \omega))^{-1} d \omega=0,
$$

we obtain the following theorem.

Theorem 4.1. For $K \geq 1$ and $\operatorname{Re} s \geq 0$,

$$
\mathbf{E} e^{-s \tau_{K}}=\left\{1-(1-f(s)) \frac{1}{2 \pi i} \int_{D_{\omega}}(1-\omega)^{-1}(\omega-f(s+\lambda-\lambda \omega))^{-1} \frac{d \omega}{\omega^{K}}\right\}^{-1} .
$$

Remarks 4.2. (i) There exist several methods to evaluate the Cauchy-type integral. A general method could be based on Faa di Bruno's formula, for example, [9, Appendix] or on series expansion techniques, for example, $[6,12]$.

(ii) Note that a probability distribution is uniquely determined by its Laplace-Stieltjestransform. Hence, $\mathbb{R}^{(K)}(\cdot)$ follows from inversion of $\mathbf{E} e^{-s \tau_{K}}$ (see forthcoming example).

(iii) It should be remarked that even the simplest case $K=1$ has several tantalizing technical applications, for example, [7]. Therefore, we consider the case $K=1$ in detail.

\section{The duplex system}

As an example, we consider the duplex system $(K=1)$ subjected to the particular case of deterministic failures, that is, let

$$
F(t) \equiv U\left(t-t_{0}\right):= \begin{cases}1, & \text { if } t \geq t_{0}>0 \\ 0, & \text { if } t<t_{0}\end{cases}
$$


Clearly, $f(s)=e^{-s t_{0}}$. By Theorem 4.1 and Cauchy's theorem, we obtain

$$
\mathbf{E} e^{-s \tau_{1}}=e^{-(s+\lambda) t_{0}}\left\{1-e^{-s t_{0}}\left(1-e^{-\lambda t_{0}}\right)\right\}^{-1},
$$

or

$$
\mathbf{E} e^{-s \tau_{1}}=\sum_{k=0}^{\infty} P_{k} e^{-s(k+1) t_{0}},
$$

where

$$
P_{k}:=e^{-\lambda t_{0}}\left(1-e^{-\lambda t_{0}}\right)^{k} ; \quad k=0,1, \ldots
$$

Hence, by inversion,

$$
\mathbb{R}^{(1)}(t)=1-\sum_{k=0}^{\infty} P_{k} U\left(t-(k+1) t_{0}\right), \quad t \geq 0 .
$$

Note that the survival function is the tail of a lattice distribution with period $t_{0}$ and with jump $P_{k}$ at $(k+1) t_{0} ; k=0,1, \ldots$

Remarks 5.1. Observe that $\mathbb{R}^{(K)}(\cdot)$ is also uniquely determined by its Laplace transform

$$
\frac{1-\mathrm{E} e^{-s \tau_{K}}}{s}=\int_{0}^{\infty} e^{-s t} \mathbb{R}^{(K)}(t) d t, \quad \operatorname{Re} s>0 .
$$

Consequently, the survival function of the $K$-system could be computed by a general numerical inversion procedure introduced by Blanc [3]. In particular, Blanc's method seems to be of interest in the case of nonrational transforms. Obviously, the case of rational transforms (related to Coxian distributions) should be handled by exact or numerical user-friendly software as proposed in the previous literature (see, e.g., [11] for a detailed application). Finally, the exact explicit survival function could also be obtained, for a particular family of nonrational transforms, by an inversion procedure based on elegant methods of complex analysis [12].

\section{Conclusion}

Our proposed $K$-system (and its dual) can be analyzed by elegant probabilistic methods based on queuing theory. The general $K$-system, subject to arbitrary lifetime and repair time distributions, invokes an open (harsh) problem in the theory of statistical reliability engineering. The analysis of renewable multiple standby systems is far from complete.

\section{References}

[1] V. Anisimov and J. Sztrik, Asymptotic analysis of some complex renewable systems operating in random environments, European J. Oper. Res. 41 (1989), no. 2, 162-168.

[2] A. Birolini, Quality and Reliability of Technical Systems, Springer, Berlin, 1994.

[3] J. P. C. Blanc, On the numerical inversion of busy-period related transforms, Oper. Res. Lett. 30 (2002), no. 1, 33-42. 
[4] P. Chandrasekhar and R. Natarajanan, A study of a n-unit system operating in a random environment, Reliab. Eng. Syst. Safety 66 (1999), no. 3, 223-226.

[5] J. W. Cohen, On the busy periods for the $M / G / 1$ queue with finite and with infinite waiting room, J. Appl. Probab. 8 (1971), 821-827.

[6] The Single Server Queue, North-Holland Series in Applied Mathematics and Mechanics, vol. 8, North-Holland Publishing, Amsterdam, 1982.

[7] D. V. Raje, R. S. Olaniya, P. D. Wakhare, and A. W. Deshpande, Availability assessment of a two-unit stand-by pumping system, Reliab. Eng. Syst. Safety 68 (2000), no. 3, 269-274.

[8] M. Shaked and J. G. Shanthikumar, Reliability and maintainability, Stochastic Models (D. P. Heyman and M. J. Sobel, eds.), Handbooks Oper. Res. Management Sci., vol. 2, NorthHolland Publishing, Amsterdam, 1990, pp. 653-713.

[9] L. Takács, Combinatorial Methods in the Theory of Stochastic Processes, John Wiley \& Sons, New York, 1967.

[10] E. J. Vanderperre, On the busy period for the $M / G / 1$ queue with finite waiting room, Rev. Française Automat. Inform. Rech. Opér. 1 (1974), 41-44.

[11] On the reliability of Gaver's parallel system sustained by a cold standby unit and attended by two repairmen, J. Oper. Res. Soc. Japan 41 (1998), no. 2, 171-180.

[12] Reliability analysis of a renewable multiple cold standby system, Oper. Res. Lett. 32 (2004), no. 3, 288-292.

E. J. Vanderperre: Ruzettelaan 183, Bus 158, 8370 Blankenberge, Belgium

Current address: Department of Quantitative Management, University of South Africa, P.O. Box 392, Pretoria 0003, South Africa

E-mail address: evanderperre@yahoo.com 


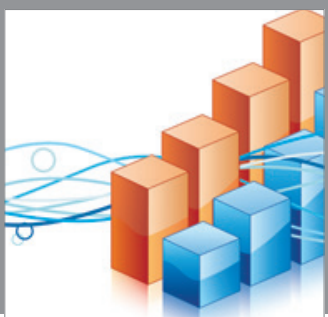

Advances in

Operations Research

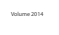

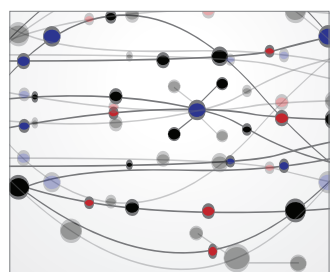

\section{The Scientific} World Journal
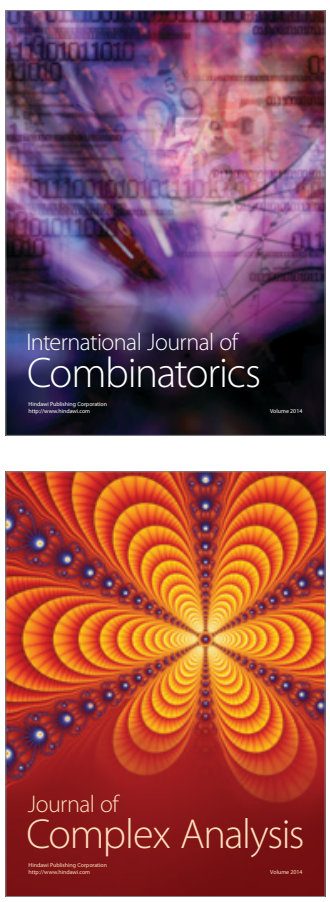

International Journal of

Mathematics and

Mathematical

Sciences
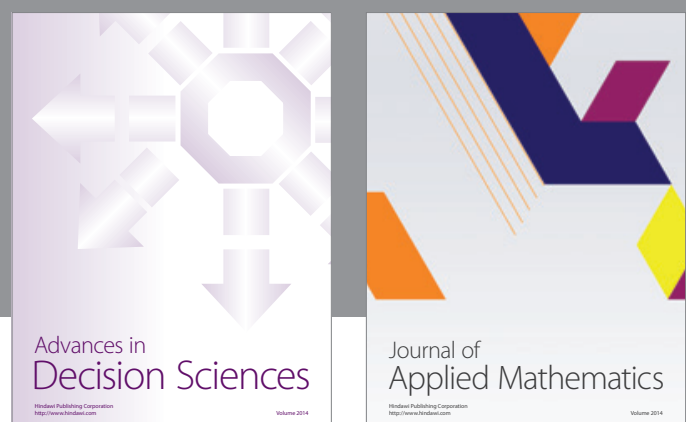

Journal of

Applied Mathematics
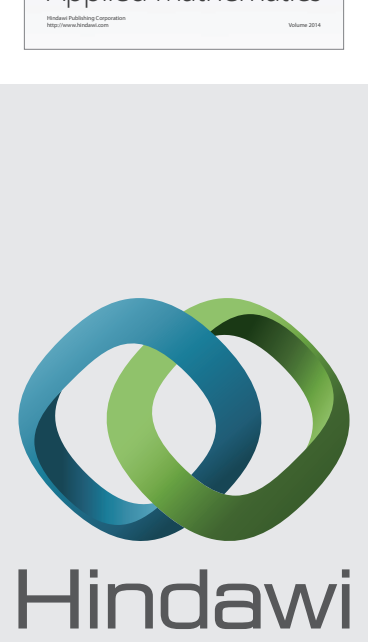

Submit your manuscripts at http://www.hindawi.com
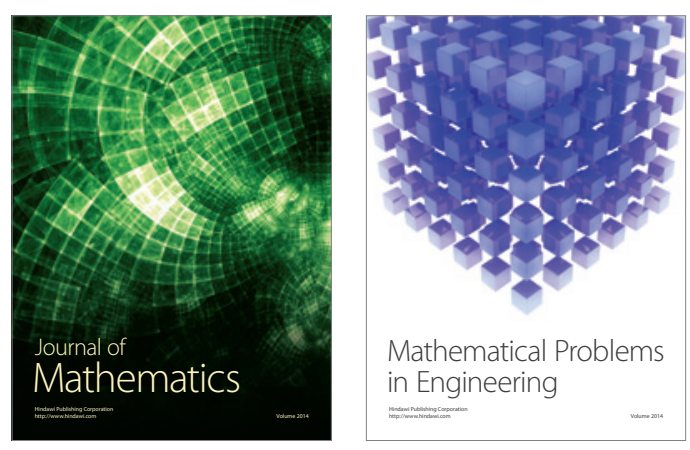

Mathematical Problems in Engineering
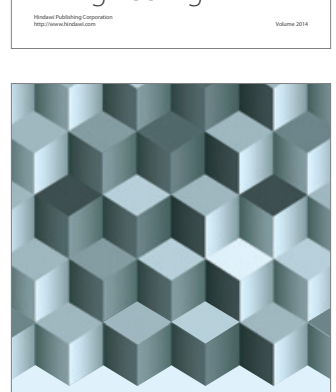

Journal of

Function Spaces
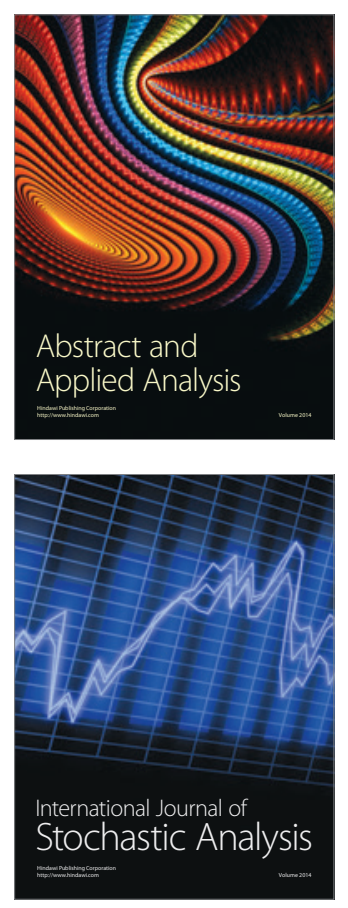

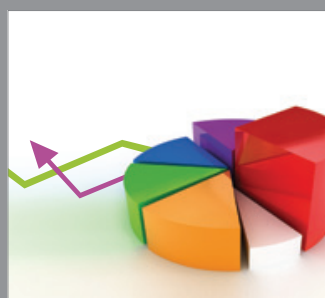

ournal of

Probability and Statistics

Promensencen
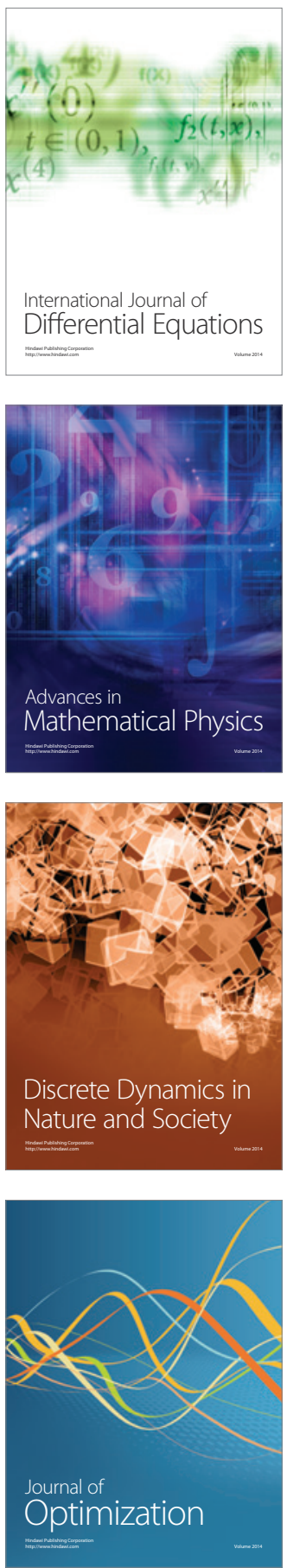